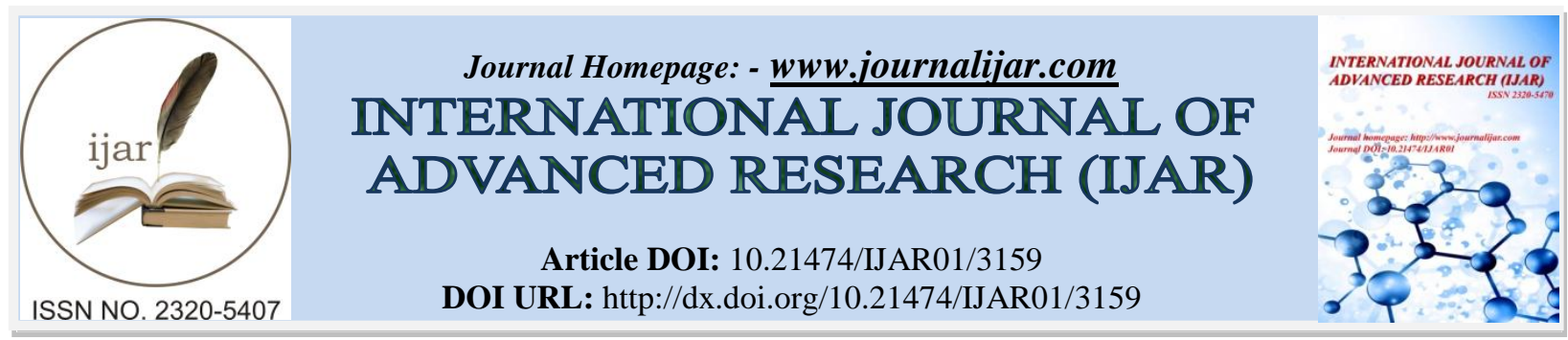

RESEARCH ARTICLE

\title{
SINGLE VISIT APEXIFICATION AND OBTURATION OF IMMATURE NECROTIC PERMANENT TEETH USING BIODENTINE - FOUR CASE REPORTS.
}

Neelanjana Majumdar ${ }^{1}$, Paromita Mazumdar ${ }^{2}$ and Utpal Kumar Das ${ }^{3}$.

1. Post Graduate student, Department of Conservative Dentistry \& Endodontics, Guru Nanak Institute of Dental Sciences and Research, 157/F Nilgunj Road, Panihati, Kolkata-700114.

2. Professor, Department of Conservative Dentistry \& Endodontics, Guru Nanak Institute of Dental Sciences and Research, 157/F Nilgunj Road, Panihati, Kolkata-700114.

3. Professor and H.O.D, Department of Conservative Dentistry \& Endodontics, Guru Nanak Institute of Dental Sciences and Research, 157/F Nilgunj Road, Panihati, Kolkata-700114.

\section{Manuscript Info}

Manuscript History

Received: 15 December 2016

Final Accepted: 10 January 2017

Published: February 2017

Key words:-

Single visit apexification, artificial

apical plug, Biodentine

\section{Abstract}

The management of a tooth with open apex consists of either the induction of a natural barrier or the creation of an artificial apical barrier. Since the conventional apexification using calcium hydroxide has certain drawbacks recent approach is to form an artificial apical plug using newer biomaterials. In addition to MTA, alternative materials are being developed and researched studied for the purpose. This article describes the successful management of traumatized and necrotic permanent maxillary incisor teeth with open apex using artificial apical barrier technique with Biodentine.

Copy Right, IJAR, 2017,. All rights reserved.

\section{Introduction:-}

Traumatic injury to an immature permanent tooth leads to loss of pulp vitality and arrested root development. Thus, endodontic management of these teeth in young patients is a great challenge. The walls are divergent and wide open apex makes debridement and obturation difficult [1]. An Apical root closure may result from apexification or bridge formation. Apexification is defined as a method to induce a calcified barrier in a root with an open apex or continued apical development of an incomplete root in a tooth with necrotic pulp [2].

Various techniques were used to induce the apexification process. The most common traditionally used medicament is Calcium Hydroxide. It was first introduced by Kaiser and Frank in 1960's. The approximate time for induction of calcified apical barrier varies between 6 months and 24 months. Although technique is efficient with predictable outcomes, it has several disadvantages like prolonged treatment time, chances of re-infection and risk of cervical fracture [3].

An alternative to apexification with calcium hydroxide is formation of an artificial apical barrier technique using MTA. Literature suggests that MTA is biocompatible with cementogenic properties and has superior sealing ability. But it has certain disadvantages like questionable antimicrobial activity, difficult to handle, potential for discolouring the tooth [4].

To overcome the disadvantages of MTA, a new calcium silicate based material; BiodentineTM (Septodont) has been introduced in 2009 claiming to be a revolutionary material capable of offering a bioactive and biocompatible 
replacement for dentine. The endodontic indications of the novel material are similar to MTA but are reported to offer several advantages including better consistency, improved handling, and quicker setting time (12 minutes) [5]. The following four case reports describe successful management of traumatized permanent anterior teeth with open apex with Biodentine apexification followed by root canal treatment followed by crown placement.

\section{Case Reports:-}

Four cases of open apices reported at different time in the Department of Conservative Dentistry and Endodontics, Guru Nanak Institute of Dental Science and Research, Kolkata- 700114 with the chief complaint of discolouration in upper front teeth region of mouth. The age of the patients were in a range between 21 to 25 years. The medical and dental histories were non-contributory. All of the four cases were presented with similar features as follows :-

History- Trauma 12-13 years back.

\section{Clinical presentation:-}

i) Discolouration

ii) Offending tooth shows negative response in electric pulp test (EPT)

iii) Non mobile and non tender

\section{Radiographic presentation:-}

Circumferential periapical radiolucency with wide open apex along with thin root dentin. The bony support of the tooth was completely intact.

\section{Treatment procedure:-}

The treatment protocol was performed as follows :-access cavity was prepared using no.2 round bur (Dentmark) and refined with endo $\mathrm{Z}$ bur (Dentsply) with rubber dam (Coltene) isolation. Working length was established by radiograph. Biomechanical preparation and circumferential filling was done with $80 \mathrm{~K}$ file. Irrigation was performed with $3 \%$ sodium hypochlorite and normal saline alternatively. Calcium hydroxide as an intracanal medicament was placed in the canal for 1 week and the access cavity was sealed with zinc oxide eugenol.

On recall visit, the tooth was found to be asymptomatic clinically and radiographically. Intracanal medicament was removed by irrigating with alternating solutions of 3\% Sodium hypochlorite and saline. The canal was completely dried with size 80 absorbent paper point and the absorbable gelatin base foam (AbGel) placed apically as a barrier.

Biodentine (Septodont) capsule was tapped on a hard surface to diffuse the apowder. After this, five drops of manufacturer's supplied liquid was dispensed into the capsule. The capsule was then placed in triturator for $30 \mathrm{~s}$. After mixing, biodentine was placed at the apical region and gently adapted to the apical portion of the canals using endodontic pluggers until an apical plug of 4-5 mm was reached. Correct placement of Biodentine apical plug was assessed radiographically. Following the placement of biodentine over the barrier, butt-end of a paper point was used to clean out any excess material from the walls. After 10-12 minutes, the hardness of the biodentine was examined using the plugger to confirm its set. Rest of the canal space was obturated with gutta flow and post obturation access cavity restoration was done with light cure composite resin (Filtek Z 250 XT, 3M ESPE) on the same day. The patients were recalled at every 1 month interval for a period of 6 months for clinical and radiographic assessment during this period.

Case 1:-

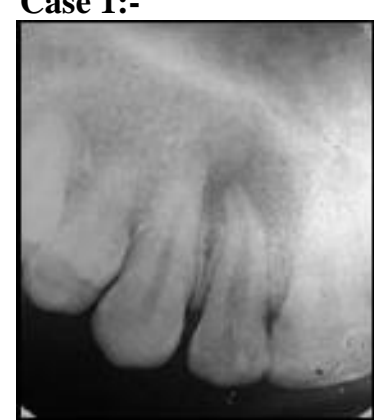

(a)

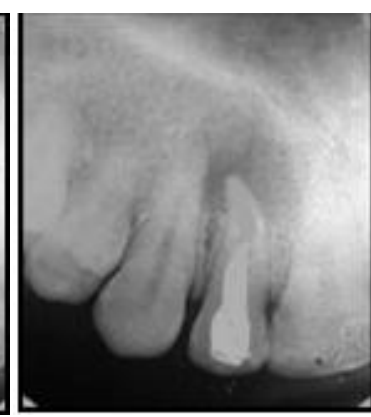

(c)

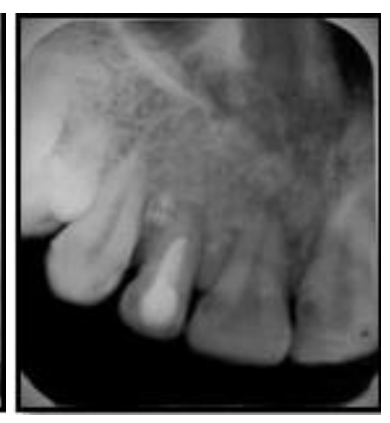

(a)- Pre operative radiograph (22)

(b)- Post operative radiograph

(c)- 6 months follow up radiograph 


\section{Case 2:-}

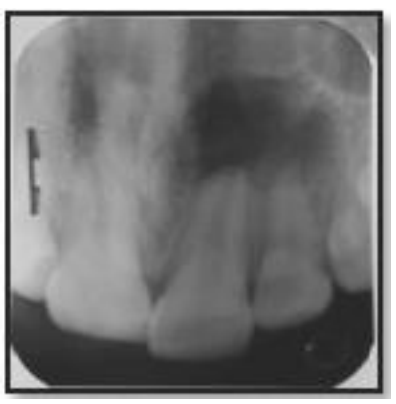

(a)

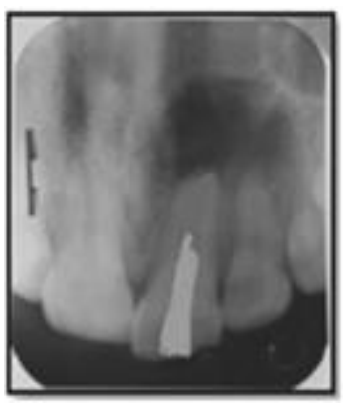

(c)

Case 3:-

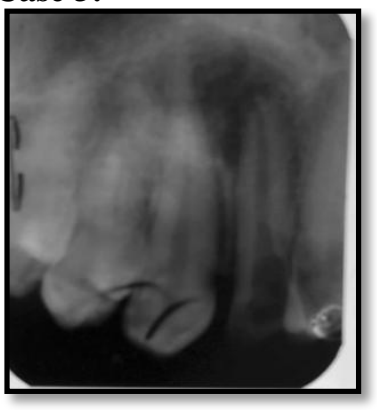

(a)

(b)
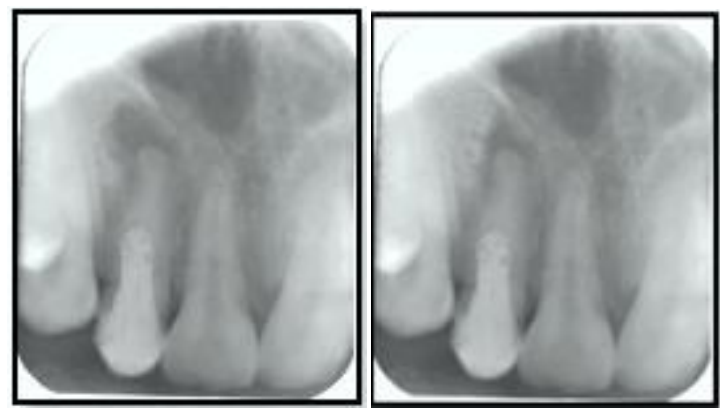

Case 4-

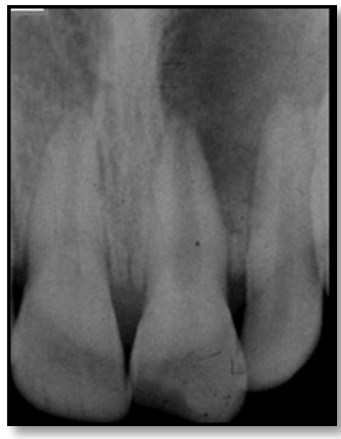

(a)
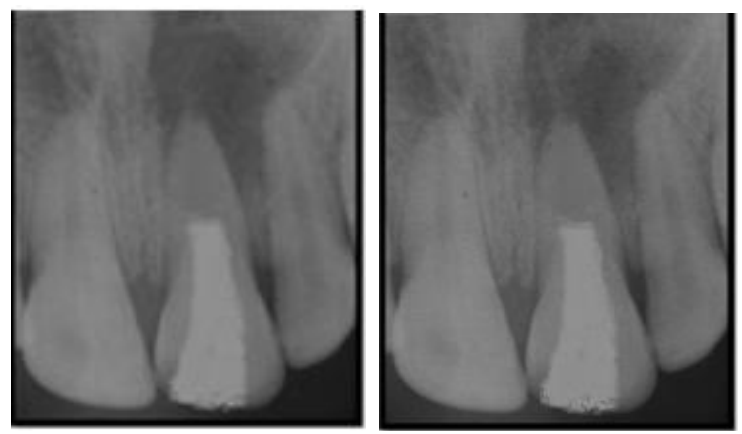

(a)- Pre operative radiograph (11)

(b)- Post operative radiograph

(c)- 6 months follow up radiograph

(a)- Pre operative radiograph (22)

(b)- Post operative radiograph

(c)- 6 months follow up radiograph (a)- Pre operative radiograph (11)

(b)- Post operative radiograph

(c)- 6 months follow up radiograph

\section{(c) -6 months follow up radiograph}

(c)

(b)

(c)

\section{Discussion:-}

Calcium hydroxide has been used in the conventional apexification technique to create an environment conducive to the formation of an apical barrier formed by osteo-cementum tissue at the end of the root canal in teeth with open apices. However, it has certain drawbacks like the long duration of therapy (3 to 21 months), susceptibility of the tooth to fracture during treatment, and susceptibility of the root canal to re-infection due to a temporary seal in the tooth [4]. With the introduction of MTA, a more convenient and less time taking technique by placing a plug of MTA in apical $4 \mathrm{~mm}$ of the root canal was conceptualized. MTA has a range of advantages such as biocompatibility, hard tissue formation, sealing ability, antibacterial property and MTA is not affected by the presence of blood [5]. But compressive strength of MTA is lower (40 MPa immediately after setting and increases to $70 \mathrm{MPa}$ after 21 days) than Biodentine (300 MPa after one month).

Biodentine is a novel innovative bioactive and biocompatible material which provides a good biological seal and excellent marginal adaptation, a high degree of biocompatibility material that was introduced by Septodont. The powder is chiefly composed of tricalcium silicate with added $\mathrm{CaCO} 3$ and zirconium oxide. The liquid portion contains calcium chloride $(\mathrm{CaCl} 2)$, as setting accelerator, in the water reducing agent. Addition of $\mathrm{CaCl} 2$ not only 
decreases setting time but also improves its handling properties. Setting time of MTA is 2 hours and 45 minutes and needs at least two appointments to complete the obturation. But the setting time of biodentine is 9-12 minutes, does not require two- step obturation and the treatment can be rendered in a single appointment. These factors reduce the chances of bacterial contamination [6]. Biodentine like MTA has an ability to initiate and continue the mineralization process. It has an elastic modulus of $22 \mathrm{GPa}$ which is very close to that of dentin 18.5GPa [7].

Many studies have been performed to demonstrate the bioactivity of Biodentine in clinical situations because it was able to stimulate initiation and development of mineralization [3]. Studies on management of large periapical lesion using Biodentine as retrograde restoration showed positive response in treatment outcome [8]. The only limitation of Biodentine is that it cannot be used in the presence of moisture unlike MTA. Hence, proper isolation is mandatory while using Biodentine. In the present case, the use of Abgel as apical matrix not only served in limiting the Biodentine to root canal but also provided an isolated environment for setting of Biodentine [9].

\section{Conclusion:-}

Biodentine, a bioactive - biomimetic material, shows promising use for apexification, and reinforcement in management of immature teeth with open apex while serving as a monoblock. However, in such cases, long term follow up is necessary to ensure and evaluate success. Periapical healing was seen on radiographic observance. This may due to the fact that biodentine has superior marginal adaptation. Periapical radiolucency was almost completely healed after 6 months follow- up. In these cases, the circumferential diameter of radiolucency was decreased

\section{References:-}

1. Felippe W, Felippe M, Rocha M. The effect of MTA on the apexification and periapical healing of teeth with incomplete root formation. Int Endod J. 2006; 39:2-9.

2. American Association of Endodontists. Glossary of Endodontic terms, 7th edition. Chicago:American Association of Endodontists; 2003.

3. Ghaeth HY, Judith C, Ahmed GMS, Saif SA, Sameer SO, George E. The effect of frequency of calcium hydroxide dressing change and various pre and inter- operative factors on the endodontic treatment of traumatized immature permanent incisors. Dent Traumatol. 2012; 28:296-301.

4. Torabinejad M, Parirokh M. Mineral trioxide aggragate: A comprehensive literature review -Part II: Leakage and Biocompatibility investigations. J Endod. 2010; 36:190- 202.

5. Septodont. Biodentine -Active Biosilicate Technology, scientific file. Saint-Maur-Des-Fosses Cedex, France: R and D Department, Septodont; 2010.

6. Deenadayalan Elumalai et al, Comparison of mineral trioxide aggregate and biodentine for management of open apices, Journal of Interdisciplinary Dentistry October 27, 2016, IP: 45.123.108.23

7. Shilpi Gupta et al, Biodentine for Apical Barrier for Immature Necrotic Permanent Teeth: Report of Cases, International Journal of Contemporary Medical Research Volume 3 | Issue 11 | November 2016 | ICV (2015): 77.83

8. Ambica Khetarpal et al, Endodontic management of open apex using Biodentine as a novel apical matrix, Indian Journal of Dental Research, 25(4), 2014

9. Deenadayalan Elumalai et al, Comparison of mineral trioxide aggregate and biodentine for management of open apices, Journal of Interdisciplinary Dentistry October 27, 2016, IP: 45.123.108.2 